\title{
Glycemic Control And Raised Adenosine Deaminase Activity In Type 2 Diabetes Mellitus
}

\author{
*Hariprasath. $\mathrm{G}^{1}$, Ananthi. ${ }^{2}$ \\ ${ }^{I}$ Ph.D Scholar, Department Of Biochemistry, Rajah Muthiah Medical College, Annamalai University, \\ Annamalai Nagar, Tamil Nadu, India. \\ ${ }^{2}$ Professor, Department Of Biochemistry, Saveetha Medical College And Research Institute, Thandalam, \\ Chennai 602105.
}

\begin{abstract}
Background: Metabolic disturbance and immunological imbalance are the key factors in the pathogenesis of Type 2 Diabetes Mellitus. In the present study we measured Plasma Adenosine Deaminase (ADA) activity, a marker of cell mediated immunity in Type 2 Diabetics with Good and Poor glycemic control.

Materials \& Methodology: The study included 25 Type 2 diabetic patients with good glycemic control $(\mathrm{HbAlc}<7), 25$ Type 2 diabetic patients with poor glycemic control (HbAlc>7) and 30 healthy controls with no history of diabetes mellitus. Fasting Blood sugar, HbAlc and Plasma ADA activity were estimated in all these subjects.

Results: Overall an elevation of plasma ADA activity was observed in Type 2 diabetic subjects as compared to controls. In particular when compared to control group, much higher ADA activity was observed in Type 2 diabetic patients with poor glycemic control than Good Glycemic control $(p<0.01)$. Significant Positive correlation was also observed between ADA \& HbAlc in both good $(r=0.67, P<0.01)$ and poor glycemic controls $(r=0.645, P<0.01)$.

Conclusion: The significant raise of ADA activity with raise in HbAlc reflects activation of cell mediated immune response as a defense mechanism owing to deterioration of glycemic control in Type 2 diabetic patients.
\end{abstract}

Keywords: Type 2 diabetes mellitus, HbAlc, ADA, Cell-mediated immunity, Fasting plasma glucose.

\section{Introduction}

Type 2 diabetes Mellitus is a chronic progressive metabolic disorder charecterised by hyperglycemia resulting from relative deficiency of insulin hormone [1]. International Diabetes Federation estimates the number of diabetics in India to be 65.1 million, compared to 50.8 million in 2010 [2]. In general patients with diabetes mellitus have an increased incidence of infections caused by fungi and mycobacteria [3]. Cell mediated immunity appears to be important in host defences against certain infections, especially those caused by fungi and mycobacteria [4]. Thus immunological disturbances in type 2 diabetes mellitus have an association with cell mediated responses and in appropriate T lymphocyte function [5]. One of the possible causes of this altered cell mediated immunity in type 2 diabetes mellitus might be due to loss of metabolic control which can affect both lymphocyte function as well as lymphocyte metabolism on cellular level [6]. The quality of metabolic control is usually assessed by the determination of $\mathrm{HbAlc}$ which reflects the cumulative glycemic history of the preceding two to three months [7].

Adenosine deaminase (ADA) is an enzyme, which converts adenosine to inosine through an irreversible deamination reaction in the purine salvage pathway [9]. The highest activity of ADA has been reported in lymphocytes and monocytes [10]. Its chief role concerns the proliferation and differentiation of lymphocytes and activation of macrophages [11]. Hence it is considered as a good marker for cell immunity [12]. It is also considered to be an important enzyme for modulating the bioactivity of insulin [13] and some studies have reported it to be a predictive marker of insulin resistance [14].

The measurement of Adenosine deaminase may be helpful in understanding the immunological alterations in type 2 diabetes Mellitus [15]. So, in this study we aimed to measure the activity of Adenosine deaminase, a marker of cell mediated immunity in type 2 diabetics with good and poor glycemic control.

\section{Materials and methods}

This Cross Sectional Study was conducted from April to September 2016 at Rajah Muthiah Medical College \& Hospital, Annamalai University. A total number of 80 subjects of age group 40-55 years participated in the study out of which 30 were healthy Controls with no history of diabetes mellitus, 25 were Type 2 Diabetics with Good glycemic control (HbA1c $\leq 7)$ and 25 were Type 2 Diabetics with Poor glycemic control (HbA1c $\geq 7)$. The study was approved by the institutional ethical committee. Informed consent was obtained 
from all the subjects. The patients and Controls voluntarily participated in this study. All the Diabetic subjects were on oral hypoglycemic drugs. The Patients who were Smokers, Known alcoholics, on insulin treatment, having complications of Diabetes Mellitus, with history of type 1 diabetes mellitus, liver diseases, lung diseases, under the treatment of immunosuppressive drugs were excluded from the study.

Biochemical Analysis: $3 \mathrm{ml}$ of venous blood was collected from the subjects after $10 \mathrm{hrs}$ of fasting. Fasting blood sugar was estimated by Glucose Oxidase-Peroxidase method, Glycated Hemoglobin was measured by Ion exchange resin method and Plasma ADA activity was measured through enzymatic method by means of Glutamate dehydrogenase coupled reaction.

Statistical analysis: Statistical analysis was carried out using SPSS 20.0 and values were expressed in mean \pm standard deviation, Statistical difference between the control and diabetic groups were carried out using Student " $\mathrm{t}$ " test and $\mathrm{p}$ value of $<0.05$ was considered to be statistically significant. Pearsons Correlation analysis was performed for correlation of $\mathrm{HbA1c}$ with ADA activity.

\section{Results}

Table 1 shows the mean Fasting, HbA1c and plasma ADA activity in healthy controls and Type 2 diabetics with good and poor glycemic control.

Table 2 shows the Correlation Statistics between Plasma ADA activity and Glycemic control in healthy controls and Type 2 Diabetics with good and poor glycemic control.

The mean age of subjects in three groups namely Controls, Type 2 diabetic with good glycemic control $\&$ Type 2 diabetic with Poor glycemic control were $46 \pm 4.4,51.25 \pm 5.5 \& 53.5 \pm 6.2$ respectively. The average level of HbAlc was $5.19 \pm 0.4 \%$ in controls, $6.37 \pm 0.37 \%$ in Type 2 Diabetic good controls and $10.05 \pm 0.89 \%$ in those with Type 2 diabetic Poor controls.

Fasting Plasma glucose was normal in controls and significantly higher in Type 2 Diabetic patients $(\mathrm{p}<0.0001)$. In addition, Type 2 diabetics with poor glycemic control had significantly higher levels of fasting plasma glucose when compared to patients with good glycemic control $(\mathrm{p}<0.0001)$. The Plasma ADA activity was significantly higher in both Type 2 Diabetic patients with good glycemic control and poor glycemic control when compared with controls $(\mathrm{p}<0.001)$. In particular significantly higher ADA activity was noted in Type 2 diabetic patients with Poor glycemic control than Good glycemic control $(\mathrm{p}<0.0001)$.

The Correlation between Plasma ADA activity and HbAlc revealed a highly significant $(p<0.01)$ positive correlation in both Type 2 Diabetics with good glycemic control $(\mathrm{r}=0.67)$ and poor glycemic control $(\mathrm{r}=0.645)$. While in control group this correlation was found to moderately positive $(\mathrm{r}=0.4032)$ with a statistical significance of $(\mathrm{p}<0.05)$.

Table 1: Comparison of fasting plasma glucose, $\mathrm{HbA} 1 \mathrm{c}$ and plasma ADA between controls and type 2 diabetic patients with good and poor glycemic control.

\begin{tabular}{|l|c|c|c|}
\hline Parameters & $\begin{array}{c}\text { Controls } \\
(\mathbf{n = 3 0})\end{array}$ & $\begin{array}{c}\text { Good glycemic } \\
\text { Control } \\
(\mathbf{n = 2 5})\end{array}$ & $\begin{array}{c}\text { Poor glycemic } \\
\text { Control } \\
(\mathbf{n = 2 5})\end{array}$ \\
\hline Fasting Plasma glucose (mg/dl) & $79.83 \pm 5.88$ & $115.96 \pm 22.55^{*}$ & $193.08 \pm 52.28^{*}$ \\
\hline HbA1c in \% & $5.19 \pm 0.4$ & $6.37 \pm 0.37^{*}$ & $10.05 \pm 0.89^{*}$ \\
\hline $\begin{array}{l}\text { Plasma Adenosine Deaminase } \\
\text { (IU/L) }\end{array}$ & $19.59 \pm 2.85$ & $27.42 \pm 2.76^{*}$ & $34.82 \pm 4.4^{*}$ \\
\hline
\end{tabular}

Values are expressed as Mean \pm Standard Deviation.

* Significantly different from controls at $\mathrm{p}<0.0001$ - extremely statistically significant.

Table 2: Correlation of plasma ADA activity with HbAlc in controls \& type 2 diabetics with good and poor Glycemic control.

\begin{tabular}{|c|c|c|c|c|}
\hline Group & $\mathbf{n}$ & p value & r value & $\begin{array}{c}\text { Coefficient of } \\
\text { determination } \\
\mathbf{r}^{\mathbf{2}}\end{array}$ \\
\hline Controls & 30 & $\mathrm{P}<0.05$ & 0.4032 & 0.1626 \\
\hline Good glycemic Control & 25 & $\mathrm{P}<0.01$ & 0.67 & 0.449 \\
\hline Poor glycemic Control & 25 & $\mathrm{P}<0.01$ & 0.645 & 0.4163 \\
\hline
\end{tabular}

$* \mathrm{P}<0.05$ is statistically significant.

$\mathrm{P}<0.01$ is statistically highly significant. 
Fig.1: Correlation between Plasma ADA level and HbA1c in Control group

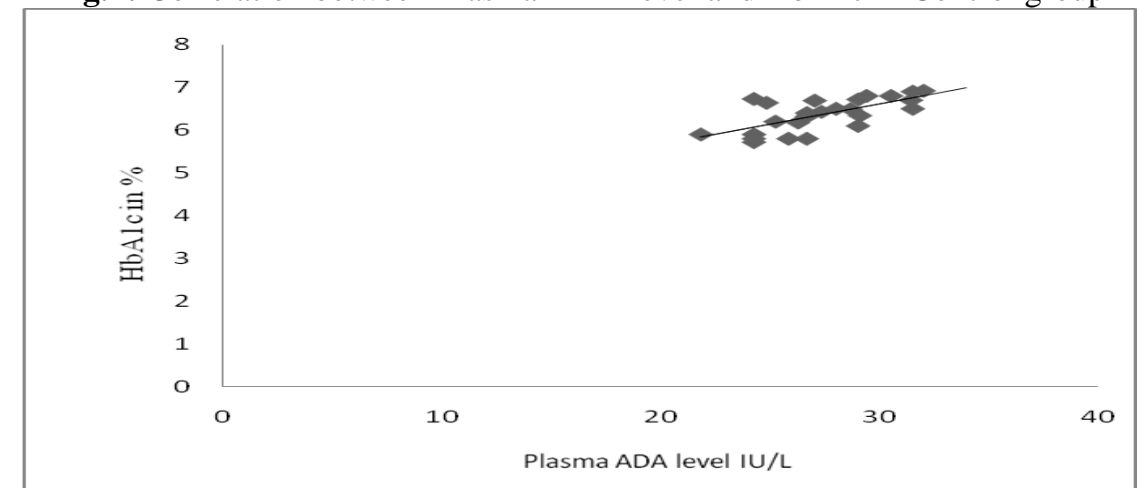

Fig 2: Correlation between Plasma ADA level and HbA1c in Type 2 Diabetic Patients with Good Glycemic Control

Fig 3: Correlation between Plasma ADA level and HbA1c in Type 2 Diabetic Patients with Poor Glycemic

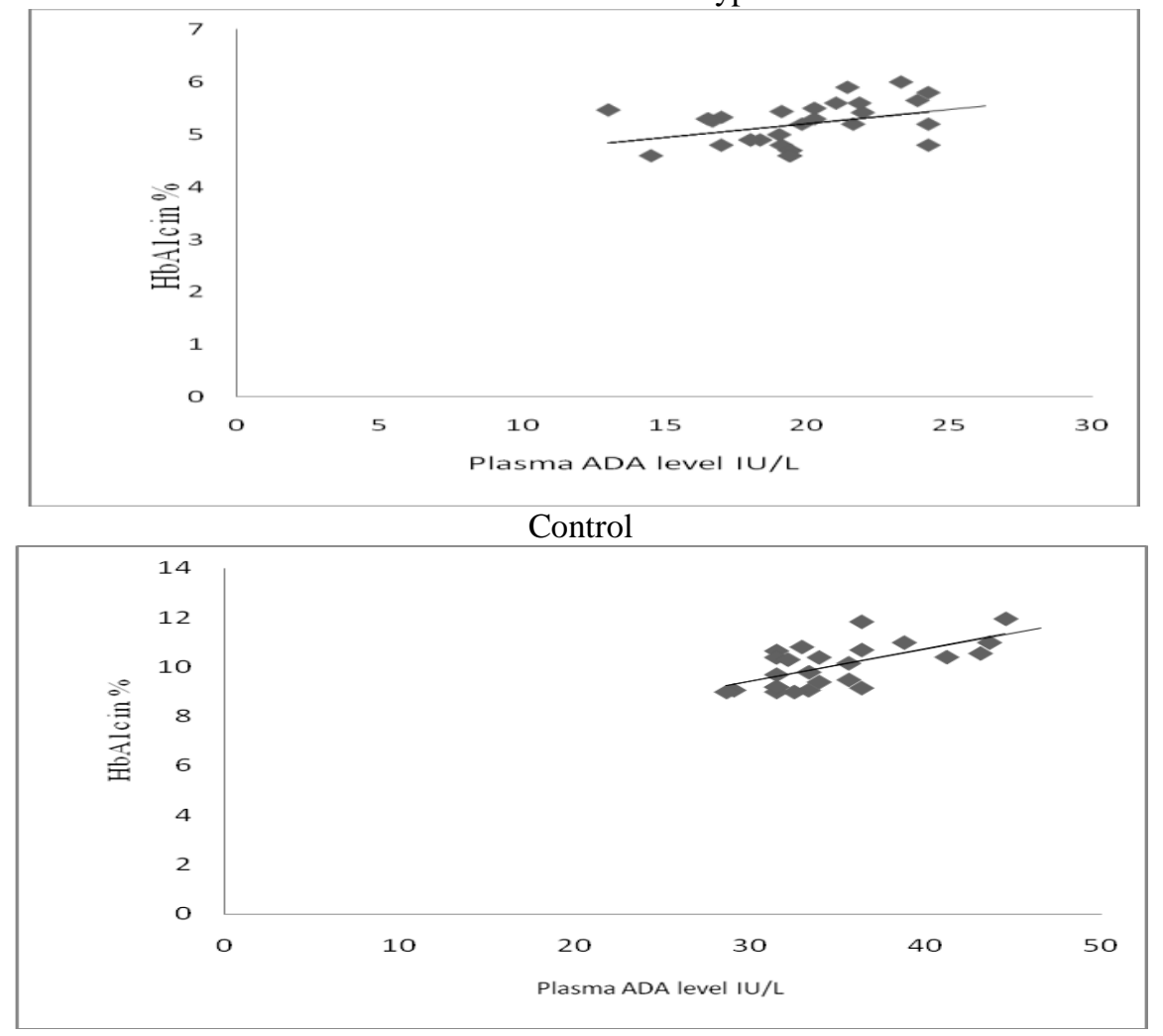

\section{Discussion}

Diabetes mellitus is a leading cause of morbidity and mortality affecting all strata of life. In this study the controls and the type 2 diabetic subjects were selected from the same age group (40-55 years) to exclude age related glucose intolerance and all the type 2 diabetic patients were on Oral hypoglycemic drug therapy. Our study assessed Plasma Adenosine deaminase activity in type 2 diabetes mellitus patients with good and poor glycemic control and also assessed the correlation between plasma Adenosine deaminase activity with glycemic control. In our study the type 2 diabetic patients were classified into two groups based on their level of HbA1c: Type 2 Diabetic patients with good glycemic control $\left(\mathrm{HbA}_{1} \mathrm{c}<7.0 \%, \mathrm{n}=25\right)$ and Type 2 Diabetic patients with poor glycemic control $\left(\mathrm{HbA}_{1} \mathrm{c} \geq 7.0 \%, \mathrm{n}=25\right)$. Thirty healthy people served as controls. The mean level of plasma adenosine deaminase in healthy controls was $19.59 \pm 2.85 \mathrm{IU} / \mathrm{L}$, which falls within the reference quoted in the Kit Methodology adopted for its analysis. The mean level of ADA quoted in the kit is <24IU/L.

In line with other studies as stated by Amandeep Kaur et al [13], Hoshino T et al [14], the level of ADA was increased significantly in Type 2 DM patients with Poor Glycemic Control (HbA1c $>7 \%$ ) with $p$ value of $<0.0001$ when compared to controls. The mean \pm SD for controls was $19.59 \pm 2.85$ and the mean \pm SD for Type 2 DM patients with Poor Glycemic control was 34.82 \pm 4.4 . 
The level of ADA was also increased significantly in type 2 DM patients with good glycemic control $(\mathrm{HbA1c}<7)$ with a $\mathrm{p}$ value of $<0.0001$ when compared to controls. The mean \pm SD for controls was $19.59 \pm 2.85$ and the mean \pm SD for type 2 DM patients with Good Glycemic control was 26.90 \pm 3.94 . But Ayse Erbagci [20] in his study reported that total ADA activity in well controlled patients was not different from control group ( $p>0.05)$.

Our Study also showed a direct relationship suggesting that strong positive correlation between plasma ADA activity and Glycated hemoglobin in both type 2 diabetics with good ( $r$ value 0.67 ) and poor glycemic controls ( $\mathrm{r}$ value 0.645$)$ and was statistically significant $(\mathrm{p}<0.01)$. Lowering or normalization of HbAlc level was associated with decreased Adenosine deaminase activity. This is in consistent with the previous studies of Kurtul et al.,[16] and Ayșe Erbagc et.al, [17].

The high total ADA activity in this study could be due to the abnormal T-lymphocyte responses or proliferation; which may point towards a mechanism releasing ADA into circulation. Insulin plays a classical role in the T-cell intermediary metabolism. It is believed that insulin enhances lymphocyte function, differentiation and proliferation and maintains the activated state of the T lymphocyte by enhancing the energy requirements and protein synthesis necessary for proper lymphocyte functioning [2]. Therefore defects in insulin action may lead to delayed responsiveness of T lymphocytes to antigens, thus high ADA activity in type 2 diabetes mellitus might be due to altered insulin related T-lymphocyte function. Cellular studies have shown that severe hyperglycemia reduces the function of immune cells and increases inflammation [18]. And also insulin may modulate its action on glucose metabolism in the tissues by changing the activity of adenosine deaminase in type 2 diabetic patients $[19,20]$.

In conclusion the significant raise of ADA activity with raise in HbAlc reflects activation of cell mediated immune response as a defense mechanism owing to deterioration of glycemic control in type 2 diabetic patients.

\section{References}

[1]. World health organization. Definition, diagnosis and classification of diabetes mellitus and its complications. Geneva: World health organization; 1999.

[2]. Shiva prakash, S.Chennaiah, YSR Murthy, E.Anjaiah, S. Ananda Rao, C.Suresh. Altered Adenosine Deaminase activity in the Type 2 Diabetes Mellitus. JIACM 2006; 7(2): 114-7.

[3]. Kirkpatrick, C.H.R.R.Rich and J.E.Bennett.1971.chronic mucocutaneous candidiasis : model building of cellular immunity. Ann.Intern.Med.74:955-978.

[4]. Thornton, G.F.19171. Infections and diabetes. Med.Clin.North Am. 55:931-938.

[5]. C.hang FY, Shaio MF. Decreased cell-mediated immunity in patients with non-insulin dependent diabetes Mellitus. Diabetes Res Clin Pract 1995; May; 28(2):137-46.

[6]. Nancie J. MacIver, ${ }^{1}$ Ryan D. Michalek, ${ }^{2}$ and Jeffrey C. Rathmel. Metbolic Regulation of T Lymphocytes. Annaual Review of Immunology Vol.31: 259-283.

[7]. Shariq I. Sherwani, Haseeb A. Khan, Aishah Ekhzaimy, Afshan Masood, and Meena K. Sakharkar. Significance of HbA1c Test in Diagnosis and Prognosis of Diabetic Patients . Biomark insights 2016; 11: 95-104.

[8]. Stentz FB ${ }^{1}$, Kitabchi AE, Activated T lymphocytes in Type 2 diabetes: implications from in vitro studies. 2003 Aug; 4(6):493-503.

[9]. Vanessa Sauer, Immacolota Brigida, Nicolo Carriglio, Alessandro Aiuti.Auto immune dysregulation and Purine Metabolism in

[10]. Adenosine Deaminase deficiency. Aisha Frontiers in Immunology. 2012; 3:265:1-19

[11]. Vineet Kumar Khemka, Debajit Bagchi, Arindam Ghosh, Oishimaya Sen,Aritri Bir, Sasanka Chakrabarti, and Anindita Banerjee. Raised Serum Adenosine Deaminase Level in Nonobese Type 2 Diabetes Mellitus. The Scientific World Journal 2013(9):404320.

[12]. Sibel Yurt, Canan Kucukergin, Burcu Arpinar Yigitbas, Sule Seckin, Huseyin Cem Tigin, and Ayse FIliz Kosar. Diagnostic utility of serum and pleural levels of adenosine deaminase , and interferon- $\gamma$ in the diagnosis of pleural tuberculosis. Multidiscip Respir Med. 2014;9(1):12.

[13]. A Sujatha Rani, H.Kishan Reddy, Prabhavati Modi.Diagnostic Value of the Ratio of Pleural Fluid Adenosine Deaminase Activity to Serum Adenosine Deaminase Activity in Children with Tuberculosis and Non Tuberculosis Pleural Effusion MRIMS JOURNAL OF HEALTH SCIENCES. 2014 ; 2(1):34 -36.

[14]. Amandeep Kaur, Sahiba Kukreja, Naresh Malhotra, Neha.Serum Adenosine Deaminase Activity and Its Correlation with Glycated Haemoglobin Levels in Patients of Type 2 Diabetes Mellitus. Journal of Clinical and Diagnostic Research .2012;6(2): 252-256.

[15]. Hoshino T, Masuoka K, Tsuboi, I, Itoh K, Nonaka K, Oizumi K. Elevated Adenosine Deaminase activity in the serum of patients with diabetes mellitus. Diabetes Res Clin Pract. 1994;25(2):97-102.

[16]. Adenosine Deaminase as marker of insulin resistance. Bhanukumar Muthiah, Adarsh L.S, Naveen Krishna Peddi, VInetha Bharathan Menon. Int J Res Med Sci 2016;4(7):2972-2979.

[17]. Kurtul N, Pence S, Akarsu E, Kocoglu H, Aksoy Y, Aksoy H. Adenosine Deaminase activity in the serum of type 2 diabetic patients.Acta Medica (Hradec Kralove) 2004; 47 (1): 33-35.

[18]. Erbagci, Mustafa Akin, Oya koyluoglu, Ayce Binnur et al., Elevated Adenosine Deaminase activity is not implicated in Microvascular complications of Type II Diabetes Mellitus except HbA1c. Turkish Journal of Endocrinology and Metabolism. 2000;4(3):95-99.

[19]. Effect of Dipeptidyl Peptidase-4 Inhibitors on Adenosine Deaminase Activity in Type-2 Diabetes Mellitus. Sumit Jhajharia, Tapaswini Pradhan,Anjana Ekka and Ashok Kumar Das. Biomedical Research 2014;25 (4): 789-793.

[20]. Immune dysfunction in patients with diabetes mellitus (DM). Suzanne E Geerlings, Andy I.M Hoepelman.FEMS Immunol Med Microbiol. 1999;(3-4):259-65.

[21]. Joanna Rutkiewicz and Jan G \& ski . On the role of insulin in regulation of adenosine deaminase activity in rat tissues. Department of Physiology, Medical School, Bialwtok, Poland. FEBS Lett. 1990;271(1-2):79-80. 